\title{
Fractura de aguja espinal. Caso reportado durante anestesia raquídea
}

\section{Spinal needle fracture. Case report during spinal anesthesia}

\author{
Dra. Arisbet Rocha-Andrade,* Dra. Verónica Jiménez-Maciel,* \\ Dr. Javier Espinosa-Barrera, ${ }^{\ddagger}$ Dra. Bertha Trejo-Ángeles ${ }^{\S}$
}

Citar como: Rocha-Andrade A, Jiménez-Maciel V, Espinosa-Barrera J, Trejo-Ángeles B. Fractura de aguja espinal. Caso reportado durante anestesia raquídea. Rev Mex Anestesiol. 2021; 44 (4): 305-310. https://dx.doi.org/10.35366/100877

RESUMEN. La anestesia raquídea o espinal es la técnica anestésica utilizada en diferentes procedimientos quirúrgicos. Para ello, existen diversos tipos de agujas espinales, las cuales tienen un impacto distinto de acuerdo con las características fisiológicas de cada paciente. La fractura de aguja espinal, si bien es muy rara, se cuenta entre los factores de riesgo que pueden desencadenar múltiples complicaciones asociadas con la anestesia raquídea. En este artículo se estudia un caso de esta índole reportado en el Hospital General «La Perla», Nezahualcóyotl, y se mencionan los pasos que se siguieron para su resolución. Finalmente, se ofrecen algunos consejos dirigidos a los lectores especializados, en caso de encontrarse ante un incidente similar.

ABSTRACT. Spinal anesthesia is the anesthesic technique of choice for different surgical procedures. There are several kinds of spinal needles, which have a different impact according to each patients physiological characteristics. Even though spinal needle fracture is very rare, it is among the risk factors that may lead to several complications associated with spinal anesthesia. In this article, we refer to a case of spinal needle fracture during an anesthesic procedure, reported at «La Perla» General Hospital, in Nezahualcoyotl, State of Mexico. Also, we mention the steps that were followed in order to solve our case. Finally, we offer some advice to the specialized readers, in case they have to face a similar incident.

\section{INTRODUCCIÓN}

$S^{e}$ e conoce como anestesia raquídea o espinal a la técnica utilizada en diversos procedimientos quirúrgicos, entre los que destacan ginecoobstetricia, ortopedia, urología y cirugía general. Este recurso consiste en la inyección de una dosis de anestésico local en el espacio subaracnoideo, a nivel de la columna lumbar. En cuanto a los instrumentos requeridos, la inyección se realiza con aguja espinal, cuyo diseño y calibre pueden variar, según convenga a cada caso quirúrgico ${ }^{(1)}$.

En la actualidad, existen dos tipos de agujas espinales usadas para realizar punciones lumbares: traumáticas (Quincke o punta de diamante, Tuohy, Levi) o atraumáticas (Greene, Whitacre o punta de lápiz, Sprotte, Polymedic, Gertie Marx, Atraucan). ${ }^{i}$ De esta clasificación, las segundas se caracterizan por contar con una punta en forma de cono circular cerrado, un perfil redondeado y una abertura lateral, la cual es mayor que el diámetro interior 27. En este sentido, la ventaja de emplear agujas atraumáticas para un procedimiento como el bloqueo epidural, radica en que la peculiaridad de su punta -cónica y elíptica- diseca las fibras de la duramadre, lo cual reduce el riesgo de perforarla accidentalmente y, con ello, evitar la fuga espontánea de líquido cefalorraquídeo (LCR); esto, a su vez, disminuye la incidencia de cefalea postpunción dural (CPPD) (2).

Ahora bien, existe una serie de factores de riesgo asociados con la posible deformación o fractura de las agujas espinales durante el bloqueo subaracnoideo. En el presente estudio, se expone el caso presentado durante la práctica y se ofrecen algunos consejos funcionales para tratar de evitar esta complicación, así como el tratamiento que se podría aplicar si se manifiesta este incidente ${ }^{(3)}$.

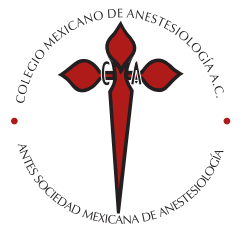

Palabras clave:

Anestesia raquídea, aguja espinal, fractura, ansiedad, obesidad, complicación anestésica.

Keywords:

Spinal anesthesia, spinal needle, fracture, anxiety, obesity, anesthesia complication.

* Anestesióloga.

‡ Residente de Urgencias.

$\S$ Jefa de Servicio de Anestesiología del Hospital General «La Perla».

Instituto de Salud del Estado de México. Nezahualcóyotl, Estado de México.

Correspondencia: Dra. Arisbet Rocha-Andrade Apaches Núm. 37, Col. San Francisco Culhuacán, Alcaldía Coyoacán, 04260, Ciudad de México, México. Tels: 55 3651-5500 y 55 5670-6099 E-mail: ary_12roa@hotmail.com

Recibido: 12-11-2019

Aceptado: 17-07-2020

Abreviaturas:

ASA = American Society of Anesthesiologists.

AN = Anestesia neuroaxial.

AINEs $=$ Antiinflamatorio no esteroideo.

$\mathrm{CPPD}=$ Cefalea postpunción dural. $\mathrm{CSE}=$ Combined spinal epidural. $\mathrm{g}=$ Gramo.

LCR = Líquido cefalorraquídeo. $\mathrm{mg}=$ Miligramo.

PDPH = Postdural

puncture headache.

PIC = Presión intracraneal.

RM = Resonancia magnética.

SNT = Síntomas neurológicos transitorios.

TC = Tomografía computada.

${ }^{\mathrm{i}}$ La aguja espinal atraumática Sprotte fue creada en Alemania en 1979 y es una modificación de la aguja espinal Whitacre 27, 28; mientras que la aguja espinal Quincke fue introducida en 1981. 


\section{REPORTE DE CASO}

Una menor de edad (17 años) con 40 semanas de gestación y un índice de masa corporal de $33.3 \mathrm{~kg} / \mathrm{m}^{2}$ fue ingresada al hospital debido a que presentaba ruptura prematura de membranas y trabajo de parto en fase activa. Con el consentimiento informado y firmado por la madre, se determinó que era urgente practicarle una cesárea bajo anestesia espinal. Según la American Society of Anesthesiologists (ASA), el riesgo clasificaba como fase II, debido a la obesidad grado I de la paciente y a que, al decir de su madre, la gestante contaba con un antecedente psiquiátrico por presentar trastorno de ansiedad generalizada, cuyo tratamiento fue suspendido por la paciente hacía un año y medio, aunque no especificó en qué consistía.

Una residente de segundo grado realizó el procedimiento de anestesia epidural previo a la cirugía, colocando a la paciente en posición de decúbito lateral izquierdo con dificultad, pues se encontraba muy ansiosa y le resultaba muy difícil seguir las indicaciones de los médicos. Se infiltró anestesia local con lidocaína 2\% 60 mg en piel y tejido celular subcutáneo. La punción lumbar se realizó con aguja hipodérmica amarilla de calibre 20 G (0.9 $\times 25 \mathrm{~mm}$ ), usando un abordaje de línea media. Se introdujo la aguja hipodérmica y, una vez colocada en la zona intervertebral L2-L3, se sumó la aguja Whitacre \#27 G corta, por medio de la técnica aguja sobre aguja. Al presentar contacto óseo, la residente procedió a reposicionar ambas agujas y, no obstante la insistencia de los médicos para que la paciente permaneciera inmóvil, le fue imposible acatar las disposiciones del personal, así como tampoco pudo evitar adoptar una postura en hiperextensión. En el momento en que se percibió un «ruido extraño», se retiraron ambas agujas; sin embargo, se observó que la aguja espinal estaba fracturada y que alrededor de $4.5 \mathrm{~cm}$ distales habían quedado incrustados dentro del espacio epidural, sin poder administrar la dosis del anestésico local, debido a la ruptura de la aguja.

\section{¿QUÉ FACTORES SE ASOCIAN A LA FRACTURA DE LA AGUJA?}

1. Posición. La posición idónea de la paciente obstétrica que requiere aplicación de anestesia neuroaxial es la de decúbito lateral izquierdo. Lo ideal es que el paciente mantenga los hombros totalmente perpendiculares al plano de la mesa de operaciones; el brazo interior debe permanecer extendido por debajo del cuerpo, mientras que el brazo exterior debe apoyarse sobre el cuerpo. De lo contrario, se modifica el plano anatómico y esto podría acarrear dificultades al momento de introducir la aguja. La cabeza debe estar alineada en relación con el tronco y flexionarse buscando que la barbilla toque ligeramente el esternón, pero sin exagerar con hiperflexiones innecesarias; lo mismo puede decirse con relación a las extremidades inferiores que tendrán que doblarse a nivel de las rodillas y luego subir los muslos acercándolos sin forzamiento hacia el abdomen para que, de esta manera, se localicen adecuadamente la línea de Tuffier e interescapular, logrando una punción óptima en las embarazadas, en L2-L3 ${ }^{(4)}$.

La disposición corporal del paciente para la administración de anestesia espinal es fundamental para que los resultados sean beneficiosos, tanto durante como después del procedimiento. Una mala postura puede causar inserciones repetidas de la aguja espinal, y con ello aumentar el riesgo de dolor de espalda y de cabeza por punción postural (PDPH), hematoma epidural e, incluso, trauma neural. Si se requieren bajos niveles de anestesia sensorial lumbar y sacra para el procedimiento quirúrgico, con frecuencia se pide a los pacientes que tomen asiento durante la aplicación. En este caso, el punto clave para realizar un bloqueo neuroaxial adecuado es reducir la lordosis lumbar, con el objetivo de facilitar el acceso al espacio intervertebral ${ }^{(4)}$.

2. Obesidad. Las diferentes técnicas neuroaxiales que se recomienda tener en cuenta para pacientes que padecen obesidad son: técnica espinal de un solo disparo, espinal continua, epidural o epidural espinal combinada (CSE). De estas alternativas, la anestesia espinal de un solo disparo proporciona un bloqueo de inicio rápido y confiable; favorece las condiciones operativas y procura serenidad al paciente, por lo que disminuye la necesidad de analgésicos suplementarios. Por sus beneficios, se sugiere en particular para atender los partos de pacientes que presenten obesidad mórbida, apnea obstructiva del sueño o vías aéreas potencialmente difíciles. A pesar de las ventajas señaladas, existen dos limitantes para el uso de la anestesia espinal de un solo disparo en pacientes con esta clase de condiciones. La primera se relaciona con la incapacidad de extender la duración del bloqueo neuroaxial. Dado que para colocar al paciente con obesidad en la mesa operatoria se requeriría de mayor tiempo, debido a la dificultad de adoptar la posición quirúrgica, se prefiere una técnica continua que asegure la posibilidad de alargar la duración del bloqueo, pues para el caso de esta población de pacientes, la duración del tiempo quirúrgico a menudo se prolonga por presentarse inconvenientes en el transoperatorio. Asimismo, de esta manera se evitan, tanto la necesidad de inducir anestesia general, como el riesgo de que la duración de la cirugía exceda la duración del bloqueo espinal.

La segunda limitante se relaciona con la facilidad para realizar el mencionado bloqueo neuroaxial. Con mayor frecuencia, las agujas espinales de punta de lápiz de calibre 25-27 se usan en la población obstétrica para disminuir el riesgo de presentar dolor de cabeza postpunción dural (PDPH). No obstante, puesto que son agujas de calibre pequeño, el introductor utilizado sería relativamente corto, lo cual podría representar un desafío técnico en el procedimiento anestésico para mujeres en labor de parto con obesidad excesiva y mórbida, puesto que el grosor del tejido en la región lumbar dificulta el acceso al espacio intervertebral. Ya que el calibre es mayor, se recomienda utilizar una aguja 
Tuohy para identificar el espacio epidural con mayor facilidad en pacientes con sobrepeso. En este caso, la aguja Tuohy más grande actuaría como un introductor ideal para la aguja espinal de calibre pequeño, lo cual permitiría la ubicación del espacio intratecal en la técnica epidural espinal combinada (CSE) de aguja a aguja. De hecho, un estudio reciente informó que se necesitan menos intentos para colocar anestesia espinal en los partos obesos mórbidos con una técnica CSE en comparación con la técnica espinal de un solo disparo ${ }^{(5)}$.

3. Ansiedad. La ansiedad es una condición patológica que se caracteriza por una sensación de miedo constante, acompañada por algunos síntomas somáticos resultantes de la hiperactividad del sistema nervioso autónomo. Se trata de un trastorno muy común entre los pacientes que han sido tratados por algún motivo en hospital, en quienes la prevalencia de esta condición es de 10-30\%. Asimismo, la ansiedad preoperatoria se ubica en una tasa de 60 y $80 \%$ en pacientes programados para cirugía, por lo que generalmente representa un factor que influye de manera desfavorable tanto en los procedimientos quirúrgicos como en la aplicación de anestesia y, en consecuencia, en la curación postoperatoria ${ }^{(6)}$. Entre las contraindicaciones relativas al bloqueo neuroaxial se encuentran las alteraciones psiquiátricas -ansiedad o angustia extrema en el paciente, así como incapacidad para comunicarse- y las deformidades anatómicas ${ }^{(7)}$.

4. Técnica. Se tiene conocimiento de varios informes de casos de deformación y fractura de agujas espinales de calibre estrecho. Las razones más comunes por las cuales estos instrumentos llegan a romperse son el impacto con el hueso o la resistencia a ligamentos/cápsulas rígidos, aun cuando no se aplica una fuerza excesiva. Entre las prácticas que pueden provocar la ruptura o la fragmentación de la aguja se cuentan, por lo menos, tres: en primer lugar, cuando se redirige la aguja sin haberla retirado a los tejidos subcutáneos superficiales; en segundo, si se retira y redirige la aguja manteniendo, al mismo tiempo, el introductor en su lugar y, en tercero, cuando se manipula la aguja después de haber sido removido el estilete. La mejor manera para evitar la fractura de la aguja cuando debe ser reubicada, es retirarla junto con el estilete al tejido superficial. La mayoría de los casos médicos que han sido publicados informan que la aguja espinal se rompe cuando se intenta retirar la aguja debilitada a través del introductor, lo cual ocasiona que éste corte el extremo distal de la aguja espinal.

En otros casos, las pruebas in vitro muestran que la aguja espinal hueca sin un estilete corre más riesgo de doblarse y romperse cuando se somete a un estrés axial excesivo. En ese sentido, mantener el estilete dentro permite que la aguja funcione como un eje de metal sólido, lo cual evita incidentes, pues la vuelve más resistente a una posible fractura. Por otra parte, el estilete también puede influir en la deflexión de la aguja espinal (en el caso de un bisel angulado). Cuando se presentan dificultades con las agujas más estrechas, se puede emplear la técnica de aguja a través de ésta. Las agujas espinales de calibre más angosto, especialmente la variedad más larga, son más difíciles de usar, sobre todo en pacientes obesos. Esta «aguja espinal a través de la técnica de aguja epidural» se puede utilizar para lograr un mayor apoyo de la aguja espinal; en otras palabras, la aguja epidural Tuohy funciona como guía para la espinal hacia la duramadre. Si el procedimiento es difícil y requiere varios intentos, se recomienda reemplazar la aguja espinal regularmente por una nueva ${ }^{(3)}$.

5. Complicaciones asociadas con la anestesia neuroaxial. Por lo general, se tiene la idea de que entre las pacientes gestantes existe un menor riesgo de presentar eventos adversos después de la anestesia neuroaxial; sin embargo, estas complicaciones pueden ocurrir en pacientes obstétricas y no-obstétricas, y su presencia dependerá tanto de la aplicación adecuada como de las condiciones médicas y de salud de los pacientes. A continuación, se presentan algunas de las complicaciones que se presentan después de un bloqueo neuroaxial ${ }^{(8)}$.

a. Cefalea postpunción dural (CPPD): es la complicación más frecuente; se presenta durante las 72 horas posteriores a una punción lumbar (terapéutica/diagnóstica) como cefalea frontal y occipital de tipo punzante y es el resultado de una fuga continua de líquido cefalorraquídeo (LCR). La presión intracraneal (PIC) cae cuando la pérdida de LCR es menor a la producción, lo cual ocasiona una dilatación compensatoria de las venas meníngeas y trae como consecuencia que el encéfalo se desplace de su posición habitual al disminuir el amortiguamiento hídrico. Asimismo, puede ocasionar alteraciones en la complianza craneoespinal (jóvenes y mujeres embarazadas son principalmente susceptibles).

b. Dolor de espalda: después de la anestesia neuroaxial (AN), presentar sensibilidad en una región localizada de la espalda es, por lo general, un padecimiento común. La mayoría de estas afecciones mejoran con el transcurso de algunos días sin convertirse en dolores crónicos; no obstante, es por esta razón que algunos pacientes rechazan la anestesia espinal en cirugías posteriores. Se cree que tal dolencia es causada por diversos factores, por un lado, debido a la lesión traumática de los ligamentos, fascia, hueso o raíces nerviosas durante el procedimiento y, por el otro, porque la relajación de los músculos paraespinales -por efecto del anestésico-suele aplanar la curvatura espinal y estirar, tanto los ligamentos paraespinales como las cápsulas articulares, causando molestia. Estas alteraciones, especialmente en posición de litotomía, predisponen a los pacientes a presentar este síntoma en el postoperatorio. El malestar leve es, usualmente, controlado con antiinflamatorios no esteroideos (AINEs), mientras que el dolor severo requiere más estudios para descartar la posibilidad de hematoma 
epidural o absceso, complicaciones que pueden requerir intervención quirúrgica.

c. Síntomas neurológicos transitorios (SNT): usualmente, los SNT se presentan con dolor y/o disestesia originada desde el área glútea que irradia a muslos posteriores. Esta sintomatología típicamente inicia entre 2 y 24 horas después de la anestesia espinal y persiste durante varios días. Puede ser aliviada con deambulación y/o fármacos antiinflamatorios no esteroideos (AINEs). Si bien ni la patogénesis de los SNT ni su impacto clínico están totalmente precisados, se sabe que la obesidad y la posición de la litotomía son factores de riesgo que pueden determinar su presencia. Asimismo, estudios previos han mostrado que el surgimiento está asociado con el uso de concentraciones altas de lidocaína (5\%).

d. Hematoma epidural: aunque suele ser una secuela que se presenta en muy pocos casos, existe la posibilidad de que la anestesia neuroaxial dé origen a un hematoma epidural, pues la punción de vasos en el plexo venoso puede generar sangrado dentro del espacio epidural con o sin coagulopatía preexistente. La acumulación de sangre en el espacio epidural incrementa la presión en la médula espinal o cauda equina y, con ello, aumenta el riesgo de isquemia o infarto. Después de la aparición brusca de síntomas como dolor de espalda, debilidad de miembros inferiores y disfunción vesical, el hematoma espinal o epidural puede permanecer silente durante un tiempo prolongado. De acuerdo con un estudio retrospectivo, la incidencia de hematoma epidural inducido por AN es aproximadamente 1/18,000 para anestesia epidural y 1/158,000 para anestesia espinal. Los factores de riesgo incluyen coagulopatía preexistente, falla renal, terapia anticoagulante, edad avanzada, osteoporosis, anormalidades espinales y el empleo de una aguja más larga de lo recomendado. Si se sospecha de la presencia de hematoma epidural, deben llevarse a cabo estudios diagnósticos de emergencia lo más pronto posible, para evitar que se desencadene un déficit neurológico permanente. De ser el caso, es imperativo la realización de un monitoreo de los pacientes por medio de la colocación permanente de un catéter epidural. Efectuar una descompresión quirúrgica dentro de las 8 horas posteriores al inicio de los primeros síntomas puede reducir la posibilidad de desarrollar discapacidad neurológica permanente.

e. Lesión directa de la médula espinal o lesión de la raíz nerviosa: en aproximadamente $80 \%$ de la población adulta, la médula espinal termina en la parte inferior de la espalda en L1, mientras que en 20\% restante, en L2. Para evitar dañar el cono medular al momento de realizar cualquier técnica neuroaxial, se recomienda permanecer por debajo del proceso espinoso L2. Aunque es común usar la línea de Tuffier -la línea imaginaria que une las dos crestas ilíacas-como punto de referencia para localizar el espacio entre L3-L4 o el proceso espinoso L4, este procedimiento no siempre es fidedigno y puede causar una lesión directa de la médula espinal debido a una mala aplicación de la aguja, catéter o inyección intraneural. Los pacientes pueden experimentar parestesias en las extremidades inferiores durante la colocación de la aguja o catéter neuroaxial, aun cuando éstas no están asociadas con secuelas neurológicas a largo plazo. Si la sintomatología persiste durante la manipulación, se recomienda parar y reposicionar la aguja/ catéter. Si bien los síntomas neurológicos a largo plazo son raros, ameritan valoración neurológica con posibles estudios radiológicos y de conducción nerviosa ${ }^{(9,10)}$.

f. Absceso epidural: se trata también de una complicación poco común, pero de carácter serio, pues existe el riesgo potencial de desarrollar una secuela neurológica debilitante permanente o incluso un desenlace fatal. El rango de incidencia varía según diversos estudios; mundialmente, se estima que los rangos de mortalidad son de 5 a $16 \%$. Bacterias y otros patógenos pueden entrar al espacio epidural y extenderse longitudinalmente, lo cual trae como resultado la inflamación y el daño espinal a causa de la presión, trombosis, obstrucción del suministro de sangre o toxinas bacterianas. Los primeros signos de absceso espinal como la fiebre y/o malestar general suelen ser inespecíficos; asimismo, la tríada clásica de fiebre, dolor espinal y deterioro neurológico también pueden ser indetectables en etapas iniciales. Por lo general, las manifestaciones clínicas desencadenan dolor grave localizado, debilidad motora, parestesia y parálisis. El mayor agente etiológico del absceso epidural es el Staphylococcus, seguido por bacilos Gram-negativos y estreptococos. El manejo usualmente incluye la eliminación o remoción del absceso -mediante aspiración, drenaje y resección quirúrgica-y la erradicación del agente causal. De igual manera, se debe administrar antibióticos empíricos, incluyendo vancomicina y cefalosporinas, tan pronto se establezca el diagnóstico.

g. Meningitis: es una complicación muy rara pero potencialmente mortal de la AN. Puede manifestarse como el resultado de utilizar equipo contaminado o de emplear una técnica aséptica inadecuada y se presenta típicamente con síntomas que incluyen fiebre, cefalea, rigidez cervical, náusea, emesis y fotofobia. Se estima que la incidencia de meningitis seguida de AN es de alrededor de 1 en 100,000 procedimientos neuroaxiales.

h. Aracnoiditis: es la reacción inflamatoria que produce edema de la capa aracnoidea del saco meníngeo después de un evento perjudicial que ocasiona fibrosis aracnoidea, adhesiones y cicatrices. La aracnoiditis se presenta con frecuencia como irritación transitoria de la raíz nerviosa, cono medular y síndrome de cauda equina, este último suele manifestarse con dolor en espalda baja, disestesia y parestesias ${ }^{(8)}$. 


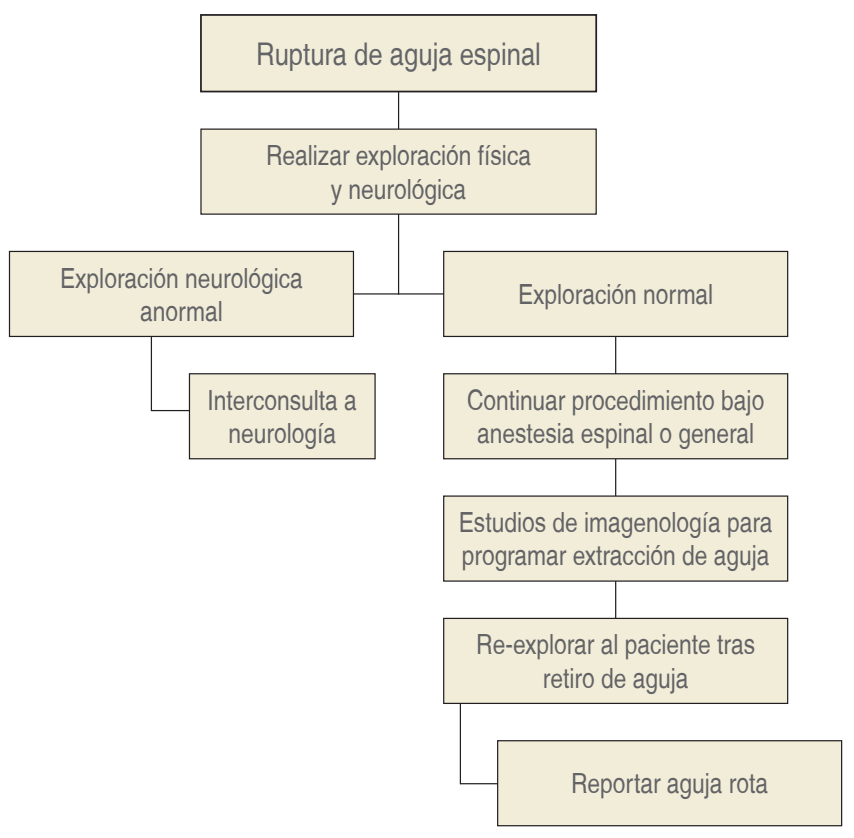

Figura 1: Algoritmo en caso de ruptura de aguja espinal.

\section{¿QUÉ HACER EN UN CASO DE RUPTURA DE AGUJA ESPINAL? (Figura 1)}

Las consecuencias de que una aguja espinal rota o algún otro «cuerpo extraño» -por ejemplo, algún resto de aguja o catéter epidural roto-quede introducido en la columna vertebral son:

1. Migración del cuerpo extraño

2. Infección o fibrosis del tejido circundante

3. Complicaciones neurológicas

En caso de que una parte de la aguja rota se encuentre en un tejido más profundo, idealmente, debe ser removida por un cirujano (espinal) o neurocirujano lo más pronto posible -de preferencia, en el mismo turno quirúrgico-al igual que se sugiere el inicio de tratamiento farmacológico para lesión traumática, que consiste en: antiinflamatorio (AINEs), esteroide y antiepiléptico ${ }^{(3)}$.

\section{¿CÓMO SE RESOLVIÓ EL CASO?}

La anestesióloga informó de inmediato a la paciente sobre el incidente que había ocurrido y marcó el área de inserción. En ese momento, la mujer no presentaba ninguno de los síntomas asociados con el fragmento de la aguja que había quedado dentro de su columna, así que se determinó no realizar un examen neurológico, ya que su diagnóstico requería practicarle la cesárea de forma urgente, y así evitar mayores complicaciones del binomio. La anestesióloga y la ginecóloga decidieron continuar con la cirugía, pero cambiaron el procedimiento inicial por la aplicación de anes- tesia general balanceada, iniciado con tratamiento antiinflamatorio no esteroideo (AINEs), metamizol 2 g en infusión, así como la administración de esteroide, dexametasona 8 mg intravenoso. Por su parte, la paciente se mostró de acuerdo con las especialistas, así que se llevó a cabo la intervención quirúrgica de cesárea y, posteriormente, se le tomó una radiografía de columna anteroposterior y lateral (Figuras 2 y 3). Debido a que el hospital donde se presentó el evento no cuenta con esos recursos, no se realizó otro tipo de estudio como la tomografía axial computarizada y la resonancia magnética. En cuanto se obtuvieron los resultados de las radiografías, se solicitó interconsulta con un médico neurocirujano, quien refirió que tenía que ser intervenida de nueva cuenta. La cirugía se programó y se efectuó de inmediato.

El neurocirujano realizó una incisión cutánea de 7 cm e identificó el fragmento de aguja rota encima del ligamento

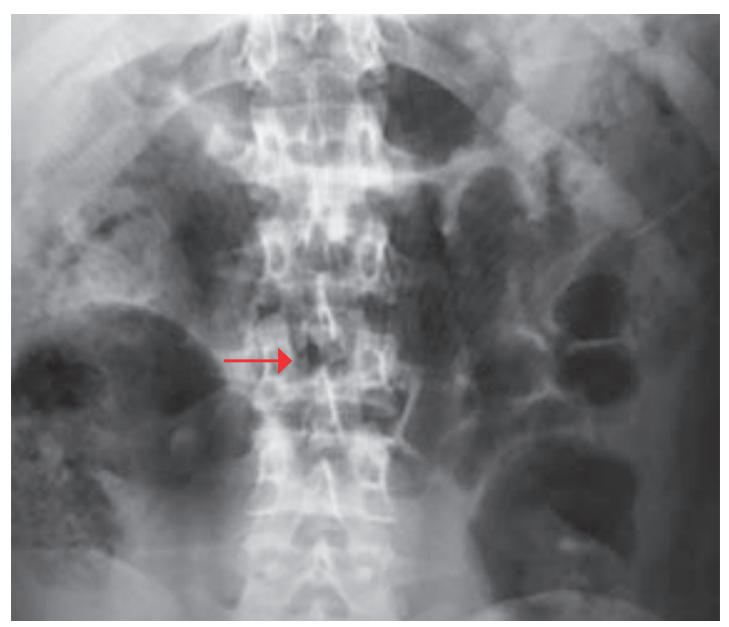

Figura 2: Visión de cuerpo extraño en radiografía anteroposterior (AP) de columna lumbar.

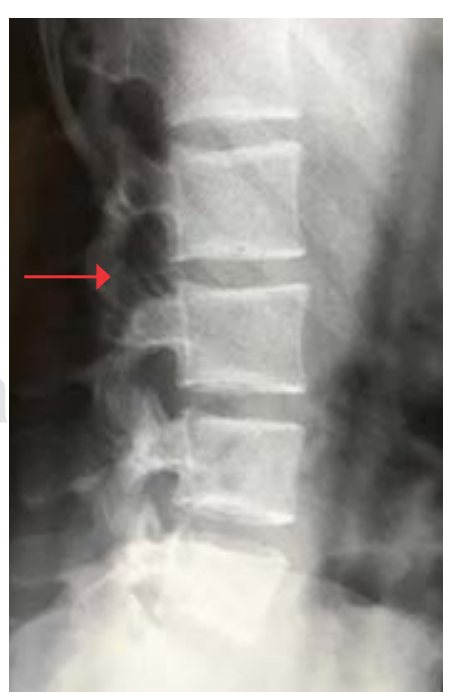

Figura 3:

Visión de cuerpo extraño en radiografía lateral de columna lumbar. 


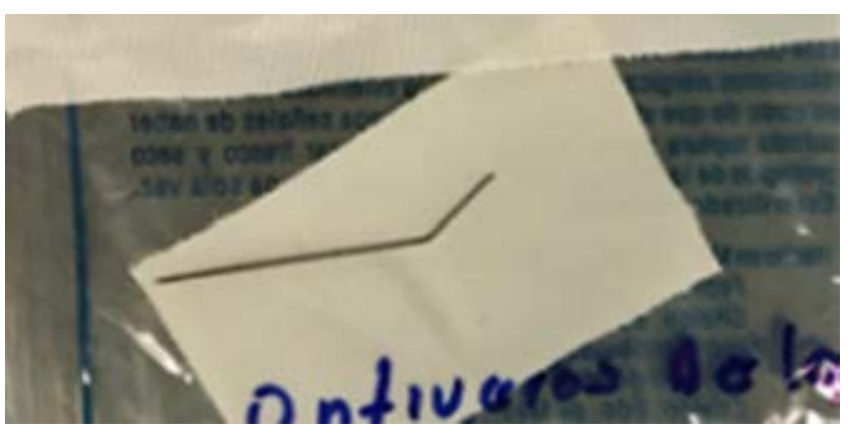

Figura 4: Cuerpo extraño (fragmento de aguja) extraído.

amarillo; en seguida, lo removió y lo extrajo (Figura 4). El procedimiento para retirar la aguja tomó alrededor de 20 minutos y durante el monitoreo transanestésico, se dio manejo a base de antiinflamatorio no esteroideo (AINEs), con la administración de 150 mg de diclofenaco intravenoso.

Aunque después de la cirugía la paciente se recuperó de forma integral sin presentar secuelas neurológicas, se decidió darle seguimiento durante los tres meses posteriores, para lo cual fue citada en un consultorio del hospital una vez por mes y se realizó de la siguiente manera:

1. Interrogatorio directo, con preguntas dirigidas tales como: ¿siente dolor, hormigueo o pérdida de la fuerza en miembros inferiores?, ¿siente dolor en la región lumbar? A lo que la paciente respondió negando estos síntomas.

2. Exploración neurológica de extremidades: se exploraron y valoraron los reflejos osteotendinosos de las extremidades inferiores con el apoyo de un martillo de reflejos.

La parte sensitiva se valoró con la paciente en decúbito dorsal sobre la mesa de exploración y con los ojos cerrados. Se pincharon suavemente ambas extremidades con un alfiler, con la finalidad de saber si percibía de la misma manera el pinchazo en ambos lados.

Estos procedimientos se siguieron en todas las citas presentes y normales; la paciente no presentó alteración neurológica de ninguna índole, por lo que no se justificó el apoyo de otro estudio de neuroimagen como la resonancia magnética (RM) o la tomografia computada (TC). Al final de las tres citas acontecidas durante estos tres meses, y a causa de una evolución favorable, el Servicio de Anestesiología decidió otorgarle el alta.

\section{CONSEJOS PARA PREVENIR RUPTURA DE AGUJA}

1. Asegúrese de que el paciente esté en una posición óptima cuando realice la inyección espinal ${ }^{(3)}$.

2. Cuando la punción espinal sea difícil, considere utilizar una aguja Tuohy para facilitar la punción intratecal y, de esta forma, evitar la fractura in situ de una aguja espinal ${ }^{(3)}$.

3. Las agujas espinales de calibre más angosto son más difíciles de emplear, en particular, para el tratamiento de pacientes obesos, por lo que se recomienda optar siempre por una técnica combinada ${ }^{(3)}$.

4. Si se percibe resistencia anormal o contacto óseo, es aconsejable no continuar con la punción, así como tampoco retirar la aguja o cambiar su dirección a través del introductor sin movilizarlo ${ }^{(11)}$.

5. Es esencial, en el caso de pacientes con riesgo de punciones difíciles, considerar el uso de ultrasonido, especialmente después de la primera punción lumbar fallida o, de igual manera, en presencia de factores de riesgo para punción lumbar difícil ${ }^{(11)}$.

6. Tome en cuenta que el antecedente de ansiedad es un factor importante que no se debe perder de vista, por lo que se sugiere premedicar al paciente y evitar que realice movimientos bruscos durante la inyección espinal.

7. En pacientes con alteraciones psiquiátricas o con suspensión de medicamentos ansiolíticos, se recomienda aplicar anestesia general balanceada para evitar complicaciones.

8. Si se produce una fractura in situ de una aguja espinal, un equipo multidisciplinario debe planificar el retiro de ésta sin demora ${ }^{(3)}$.

9. La literatura actual que describe la fractura de agujas espinales estima que es probable que el paciente permanezca libre de síntomas siempre y cuando se realice la extracción de la aguja rápidamente ${ }^{(3)}$.

\section{REFERENCIAS}

1. Luna CA, Tulcán-Toro R, Romero F, Luna MF. Medición del volumen residual en las agujas espinales tras anestesia raquídea. Rev Colomb Anestesiol. 2017;45:12-15.

2. Rodríguez-Márquez IA, Saab-Ortega N. Actualización sobre factores de riesgo para cefalea pospunción dural. MÉD UIS. 2015;28:345-352.

3. Moll A y Filippini G. Reporte de caso: ruptura de aguja espinal. Anesthesia Tutorial of the Week. 2018;378:1-5.

4. Marrón-Peña GM. Eventos adversos de la anestesia neuroaxial ¿Qué hacer cuando se presentan? Rev Mex Anest. 2007;30:357-375.

5. Lamon AM, Habib AS. Managing anesthesia for cesarean section in obese patients: current perspectives. Local Reg Anesth. 2016;9:45-57.

6. Mingir T, Ervatan Z, Turgut N. Spinal anaesthesia and perioperative anxiety. Turk J Anaesthesiol Reanim. 2014;42:190-195.
7. Rebollo-Manrique RE. Bloqueo subaracnoideo: una técnica para siempre. Rev Mex Anest. 2013;36:145-149.

8. Liu H, Brown M, Sun L, Patel SP, Li J, Cornett EM, et al. Complications and liability related to regional and neuraxial anesthesia. Best Pract Res Clin Anaesthesiol. 2019;33:487-497.

9. Pages-Arroyo E, Pian-Smith M. Neurologic complications of neuraxial anesthesia. Anesthesiology Clin. 2013;31:571-594.

10. Shiratori T, Hotta K, Satoh M, Kondo N, Ikeda J, Sasao S. A case of spinal myoclonus in a patient with elective cesarean section. JA Clin Rep. 2018;4:47.

11. Kaboré RAF, Traore IA, Traore SIS, Bougouma CTHW, Augustin P, Ouro-Bang'na Maman AF. Broken needle during spinal anesthesia: an avoidable complication. Local Reg Anesth. 2018;11:111-113. 